\title{
Long-term effects of cemented
} and cementless fixations of total knee arthroplasty: a meta-analysis and systematic review of randomized controlled trials

\author{
Cheng Chen ${ }^{1 \dagger}$, Yanyan Shi ${ }^{2 \dagger}$, Zhanpo $\mathrm{Wu}^{1 *}$, Zengxin Gao ${ }^{{ }^{*}}$, Youmin Chen ${ }^{1}$, Changzheng Guo ${ }^{1}$ and \\ Xianguo Bao ${ }^{1}$
}

\begin{abstract}
Background: To determine the long-term effects (a minimum follow-up time 8.8 years) of cemented and cementless fixations used for total knee arthroplasty (TKA).

Methods: PubMed, EMBASE, Ovid, Cochrane Library, CINAHL, China National Knowledge Infrastructure and China Wangfang database were interrogated for appropriate randomized controlled trials (RCTs) through July 2020. Data were extracted and assessed for accuracy by 2 of the authors acting independently. Any controversial discrepancies were resolved after discussion with a third author.

Result: Eight RCTs were included with low to moderate bias risks. The cemented fixation of TKA was comparable to cementless fixation in terms of implant survival (relative risk, $1.016 ; 95 \% \mathrm{Cl} 0.978$ to $1.056 ; P=0.417$ ), Knee Society (KS) knee score (standardized mean difference (SMD), $-0.107 ; 95 \% \mathrm{Cl}-0.259$ to $0.045 ; P=0.168$ ), KS function score ( $\mathrm{SMD}-0.065 ; 95 \% \mathrm{Cl}-0.238$ to $0.109 ; P=0.463)$, KS pain score (SMD $-0.300 ; 95 \% \mathrm{Cl}-0.641$ to $0.042 ; P=0.085$ ), Western Ontario and McMaster Universities Osteoarthritis Index (WOMAC) score (SMD $-0.117 ; 95 \% \mathrm{Cl}-0.307$ to $0.073 ; P=0.227)$, HSS score (SMD $-0.027 ; 95 \% \mathrm{Cl}-0.270$ to $0.217 ; P=0.829)$, range of motion (SMD $0.061 ; 95 \%$ $\mathrm{Cl}-0.205$ to $0.327 ; P=0.652$ ) at $\geq 8.8$ years of follow-up. In terms of radiographic outcomes at $\geq 8.8$ years of follow-up, the incidence of a radiolucent line in the cementless group was lower than for the cemented group (SMD 3.828; 95\% $\mathrm{Cl} 2.228$ to $6.576 ; P<0.001)$. However, the maximum total point motion (MTPM) of the cementless group was greater than for the cemented group (SMD $-0.739 ; 95 \% \mathrm{Cl}-1.474$ to $-0.005 ; P=0.048)$.

Conclusions: Long-term follow-up verified that cementless and cemented fixation have similar prosthesis survival rates, clinical scores and mobility. However, radiography suggested that each technique had an advantage with regard to the radiolucent line and MTPM.
\end{abstract}

Keywords: Total knee arthroplasty, Cemented fixation, Prosthesis, Point motion

*Correspondence: wuzp99922@sina.com; 552366015@qq.com ${ }^{\dagger}$ Cheng Chen and Yanyan Shi contributed equally to this work ${ }^{1}$ Department of Orthopedics, Nanjing Lishui People's Hospital, Zhongda Hospital Lishui Branch, Southeast University, No. 86 Chongwen Road, Lishui District, Nanjing 211200, China

Full list of author information is available at the end of the article

\section{Background}

At present, total knee arthroplasty (TKA) is the recognized effective treatment for knee osteoarthritis (OA) and rheumatoid arthritis (RA). TKA can effectively relieve pain and improve knee function, thus improving the patient's a quality of life [1]. The fixation of a knee permits use, sharing, adaptation, distribution and reproduction in any medium or format, as long as you give appropriate credit to the original author(s) and the source, provide a link to the Creative Commons licence, and indicate if changes were made. The images or other third party material in this article are included in the article's Creative Commons licence, unless indicated otherwise in a credit line to the material. If material is not included in the article's Creative Commons licence and your intended use is not permitted by statutory regulation or exceeds the permitted use, you will need to obtain permission directly from the copyright holder. To view a copy of this licence, visit http://creativecommons.org/licenses/by/4.0/. The Creative Commons Public Domain Dedication waiver (http://creativeco mmons.org/publicdomain/zero/1.0/) applies to the data made available in this article, unless otherwise stated in a credit line to the data. 
prosthesis is either cemented or non-cemented. It has been reported that $95.2 \%$ of total knee replacements have used cement for prosthesis fixation [2], and the application of cement prosthesis in clinical practice is considered to be the gold standard for TKA prosthesis fixation [3]. However, due to certain risks, such as mechanical trauma stimulation of foreign bodies during cement filling, fatal pulmonary embolism caused by the entry of small molecules of cement into the blood, and early loosening of the interface between bone and cement, there is grave concern about the long-term durability of cemented fixations. It has been suggested that a cementless prosthesis is used to replace the original cement fixation [4].

In recent years, the design concept and materials used to fabricate a cementless prosthesis have been greatly improved. Bioactive hydroxyapatite (HA) is attached to the prosthesis as a novel coating, increasing the contact surface between the implant and bone and becoming an attachment point for cells and fibrous membrane growth, which is conducive to bone growth in and around the prosthesis implant [5]. In terms of prosthetic metal materials, the trabecular metal prosthesis, which has the same elastic modulus as human bone tissue, features a multipore design to provide a more suitable biological environment for bone tissue growth [6]. Through stereo imaging analysis, it has been found that the new cementless prosthesis is superior to the traditional press fit design and cemented prostheses in reducing micro-motion, and it has been predicted that the risk of aseptic loosening will be greatly reduced [7].

The two fixation methods have their own advantages and disadvantages. The one which produces the longest prosthesis survival rate and better functional recovery is of great interest to clinical investigators. Currently, the published meta-analysis is mainly aimed at comparing the short and median-term effects of the 2 fixation methods. A meta-analysis [8] including 7 randomized controlled trials (RCTs) and quasi-RCTs with an average follow-up time of 7.1 years (range 2 to 16.6 years) found that the survival rate and clinical efficacy of the prosthesis was similar to complete cementless and cemented fixation. Similar revision rates and knee function improvements were also observed between the two fixation types in a meta-analysis [9] including RCTs with an average follow-up time of 8.4 years (range 2.0 to 16.6 years). In young patients ( $\leq 65$ years old), cementless TKA was more effective regarding radiological [10] and clinical outcomes [11] compared to cemented TKAs. A meta-analysis [12] of 17 observational studies of individuals that had revision TKA, with $\geq 24$-months follow-ups, found that there was no significant difference in failure, reoperation, aseptic loosening and infection between the cemented and non-cemented groups.
With the cohort of patients undergoing TKA becoming younger, so the demand for a longer survival rate of prostheses has increased [13]. The question of cemented or cementless fixation has once again become a hot topic of debate. It is known that the survival rate of a prosthesis is the most important indicator when evaluating the quality of fixation and is greatly related to the follow-up time. Therefore, RCTs with long-term follow-up were analyzed to compare the long-term efficacy of the 2 fixation methods in terms of implant survival rate, clinical scores and radiographic indicators, so as to provide a useful reference for the selection of a TKA prosthesis.

\section{Materials and methods}

This systematic review and meta-analysis were conducted in accordance with PRISMA guidelines.

\section{Literature retrieval strategy}

The Cochrane Library, PubMed, EMBASE, Ovid, CINAHL, China National Knowledge Infrastructure and China Wanfang databases were electronically interrogated for appropriate RCT studies on cemented and cementless fixations of TKA from the initial establishment of the database through July 2020. At the same time, the references of the included literatures were reviewed to ensure the integrity of the retrieved literature. The search terms were: total knee arthroplasty, TKA, total knee replacement, TKR, knee arthroplasty, knee replacement, cemented, cement, uncemented, uncement, cementless, randomized, randomized controlled trial and RCT. The specific Medline retrieval strategy is illustrated in Additional file 1: Table S1.

\section{Inclusion and exclusion criteria}

Participants: Patients with OA or RA of the knee who underwent TKA for the first time, regardless of age, race or nationality.

Interventions: the cementless group of patients were treated with cementless fixations (cementless femur component or cementless tibia component or both), while the cemented group were treated with cemented fixations (cemented femur component or cemented tibia component or both).

Outcomes: (1) Clinical indicators and scores: long-term survival rate of prosthesis (with any reason for revision as endpoint); Knee Society (KS) knee scores; KS functional scores; KS pain scores; Western Ontario and McMaster Universities Osteoarthritis Index (WOMAC) scores; Hospital for Special Surgery (HSS) scores; range of motion (ROM); (2) Radiographic indicators: Incidence of radiolucent line; maximum total point motion (MTPM). Outcome indicators were measured at the last follow-up. 
The included studies report at least one of the above indicators.

Study design: RCTs published only in Chinese or English.

Exclusion criteria: (1) for repeated published literatures, only the most complete or latest study were included; (2) the original literature data were not complete and could not be analyzed; (3) obvious preoperative ligament dysfunction or knee extension device dysfunction; (4) knee joint neuromuscular disease; (5) non-RCT study.

\section{Screening of literature and extraction of data}

Two authors independently carried out literature screening and data extraction strictly following the defined inclusion/exclusion criteria, and also cross-checked the literature data. If there was any difference in their point of view, one author was consulted to assist judgment until a consensus was reached. The literature screening process was divided into primary and full-text screening, respectively.

\section{Preliminary screening}

By reading the title and abstract of the article, a judgement was made of its relevance to our research question; if it was closely related to our research topic, full-text screening was carried out.

\section{Full text screening}

We carefully read the full text of the literature, extracted relevant information and included studies that met our inclusion criteria. The relevant information of the literature was uniformly extracted into an Excel spreadsheet. The extracted information mainly included: (1) Basic information of the included research; title; first author name; year of publication; email address of the corresponding author; etc. (2) Information on the prosthesis used in each study: manufacturer; patella was resurfaced or not; posterior cruciate ligament (PCL)-retaining (Yes/No); the tibial component was fixed with or without screws; bearing type; etc. (3) Baseline information: patient age; gender; inclusion and exclusion criteria; intervention measures; etc. (4) Outcome information: follow-up time; number of knees followed; prosthesis survival rate; clinical scores; radiographic indicators; etc.

\section{Bias risk assessment of the included studies}

Cheng Chen and Yanyan Shi independently evaluated the risk of bias in the included studies and cross-checked it. In case of disagreement, Zhanpo Wu was consulted to assist in the judgment until consensus was reached. Bias risk assessment software (Cochrane Collaboration Network) was employed to evaluate the bias risk of the RCTs including random methods, allocation concealment, blind implementation, complete data, selective reporting and other potential biases.

\section{Statistical analysis}

Stata 13.0 software was used for all data analysis. The standardized mean difference (SMD) and the relative risk (RR) were used as effect analysis statistics (95\% CI) for continuous variables and binary variables, respectively. A $\chi^{2}$ test was employed to analyze heterogeneity (test level set to $\alpha=0.1$ ), and the $I^{2}$ was used to quantitatively judge the heterogeneity. If $I^{2}$ was $<50(P<0.1)$ a fixed effect model was employed and if $I^{2}$ was $>50(P>0.1)$ a random effect model was used. Analysis of subgroups for sensitivity analysis was employed to address any obvious clinical heterogeneity. A $P$-value $<0.05$ was deemed to be a significant difference.

\section{Results}

\section{Flow of the study}

530 articles were retrieved from the searched databases (vide supra) using keywords related to cemented or cementless fixations of TKA, of which 167 duplicates were deleted. The title and abstract of each retrieved paper were carefully examined and those that were not related to the study topic deleted, with 22 articles remaining for full text analysis $[1-8,10,12-24]$. Six articles were excluded because the mean follow-up time was $<8$ years, 3 study without outcomes of interest, 2 studies that were not comparisons of cemented and cementless fixation, 2 studies that investigated patients receiving unicompartmental knee arthroplasty and 1 study whose design was not an RCT. Finally, a total of 8 RCTs were included in our analysis (Fig. 1).

\section{Characteristics of the RCTs analyzed}

1,123 patients (age range 41-90 years) were enrolled from 8 RCTs [25-32] conducted between 2001 and 2020. After over 8.8 years of follow-up (range 8.8-16.6 years), 984 knees at the last follow-up were included. We synthesized the evidence and assessed the long-term survivorship, clinical outcomes (KS knee scores, KS function scores, KS pain scores, WOMAC scores, HSS scores, $\mathrm{ROM}$ (degree)) and radiographic outcomes (incidence of radiolucent line, MTPM) by meta-analyses. Table 1 lists the main characteristics of patients in the included RCTs.

\section{Detailed information of total knee prostheses}

A NexGen (Zimmer, Warsaw, US) prosthesis was used in 4 RCTs, a P.F.C. Sigma (Depuy, Warsaw, US) prosthesis in 2 RCTs, 1 RCT used an Interax (Howmedica, Rutherford, US) prosthesis and 1 study an HLS Noetos (Tornier, StIsmier, France) prosthesis. All prostheses were fabricated 
Table 1 Characteristics of included RCTs $(n=8)$

\begin{tabular}{|c|c|c|c|c|c|c|c|c|}
\hline Study & Year & Country & $\begin{array}{l}\text { Time period } \\
\text { of patient } \\
\text { inclusion }\end{array}$ & $\begin{array}{l}\text { No. of } \\
\text { participants } \\
(\mathrm{C} / \mathrm{U})\end{array}$ & No. of knees ${ }^{a}(C / U)$ & $\begin{array}{l}\text { Mean age (range, } \\
\text { years) }\end{array}$ & $\begin{array}{l}\text { Mean } \\
\text { follow-up } \\
\text { time (years) }\end{array}$ & Main outcomes $^{b}$ \\
\hline Parker & 2001 & Canada & 1987.1-1988.12 & $48 / 52$ & $31 / 36$ & $66.7(50.6-76)$ & 12.8 & 1 \\
\hline Baker & 2007 & UK & 1987.6-1997.2 & 219/177 & $151 / 118$ & $70.5(41-88)$ & 8.8 & 1 \\
\hline Park & 2011 & Korea & 1997.1-1998.2 & $53 / 53$ & $50 / 50$ & $58.4(51-67)$ & 13.6 & $1,2,3,4,5,7,8$ \\
\hline Pijls & 2012 & Netherlands & 1993-1998 & 48 (total) & $24 / 24+20^{c}$ & $65.71(N R)$ & $11-16$ (range) & $2,3,6,7,8,9$ \\
\hline Choy & 2014 & Korea & $2002.1-2004.10$ & $67 / 65$ & $86 / 82$ & $67.8(49-80)$ & 9.5 & $1,2,3,5,6,7,8$ \\
\hline Kim & 2014 & Korea & 1995.1-1996.3 & $85 / 85$ & $80 / 80$ & $54.3(49-55)$ & 16.6 & $1,2,5,7,8$ \\
\hline Henricson & 2018 & Sweden & 2003-2004 & $22 / 19$ & $18 / 16$ & $55(33-59)$ & 10 & $2,3,4,7,9$ \\
\hline Batailler & 2020 & France & 2004-2005 & $65 / 65$ & $59 / 59$ & $72(50-90)$ & 13 & $1,2,3,7,8$ \\
\hline
\end{tabular}

1, Survivorship; 2, Knee Society knee score; 3, Knee Society function score; 4, Knee Society pain score; 5, Western Ontario and McMaster Universities Osteoarthritis Index (WOMAC) score; 6 , HSS score; 7, Range of motion (in degree); 8, Incidence of radiolucent line; 9 , maximum total point motion (MTPM)

$C$, cemented group; $U$, uncemented group; NR, not reported

${ }^{\text {a }}$ At the last follow-up

${ }^{\mathrm{b}}$ Included in the meta-analysis

'Cemented/HA-coated/uncoated tibial components (24/24/20)

to retain the posterior cruciate ligament with a fixed tibial structure. Three studies were concerned with resurfacing of the patella. None of the studies used screws to assist fixation. Four studies compared full-cemented with full-cementless TKA, 2 studies compared uncemented with cemented tibial component (with uncemented femoral component in both groups), 1 study compared uncemented with cemented femoral component (with cemented tibial component in both groups), and 1 study compared cemented with uncemented tibial component without reporting the condition of femoral component (Table 2).

\section{Quality of the RCTs}

The bias risk plot and summary of the data are shown in Fig. 2A, B. Three of 8 studies analyzed random sequence generation, 5 allocation concealment, 4 blinding of patients and healthcare staff and 3 blinding of outcome assessments. All 8 studies had a low risk of attribution and reporting bias. Overall, the quality of the included studies varied from average to high quality.

\section{Publication bias}

The Egger test (Additional file 1: Figs. S1-S8) did not reveal any evidence for publication bias regarding implant survival $(P=0.140)$, KS knee scores $(P=0.130)$, KS function scores $(P=0.399)$, WOMAC scores $(P=0.908)$, HSS scores $(P=0.800)$, ROM $(P=0.704)$, the incidence of the radiolucent line $(P=0.051)$ or MTPM $(P=0.939)$. The test results of KS pain scores were not generally available because only 2 studies were included in the meta-analysis.

\section{Primary outcome Implant survival}

We assessed the implant survival of cemented $(n=457)$ and cementless $(n=425)$ fixation of TKA by grouping together the survivorship at the last follow-up in 6 studies [25-27, 29, 30, 32]. After using a fixed effect model $\left(I^{2}=0 \%\right)$, the pooled data revealed the survivorship of the cementless fixation group of patients at a minimum of 8 years follow-up that was comparable to the cemented fixation group (RR, 1.016; 95\% CI 0.978 to $1.056 ; P=0.417$ ) (Fig. 3).

\section{Secondary outcomes Clinical outcomes}

KS knee scores We assessed the KS knee score of cemented $(n=298)$ and cementless $(n=317)$ fixation of TKA by grouping together the KS knee score at last follow-up in 6 studies [27-32]. After applying a fixed effect model $\left(I^{2}=41.8 \%\right)$, the pooled data indicated the KS knee score of cementless fixation group at minimum 8.8 years of follow-up is comparable to that of cemented fixation group (SMD $-0.107 ; 95 \% \mathrm{CI}-0.259$ to $0.045 ; P=0.168$ ) (Fig. 4A).

KS function score We assessed the KS function score of cemented $(n=218)$ and cementless $(n=237)$ fixation of TKA by grouping together the KS function score at last follow-up in 5 studies [27-29, 31, 32]. After employing the fixed effect model $\left(I^{2}=0 \%\right)$, pooled data analysis revealed that the KS knee scores of the cementless fixation group at a minimum 8.8 years follow-up were com- 


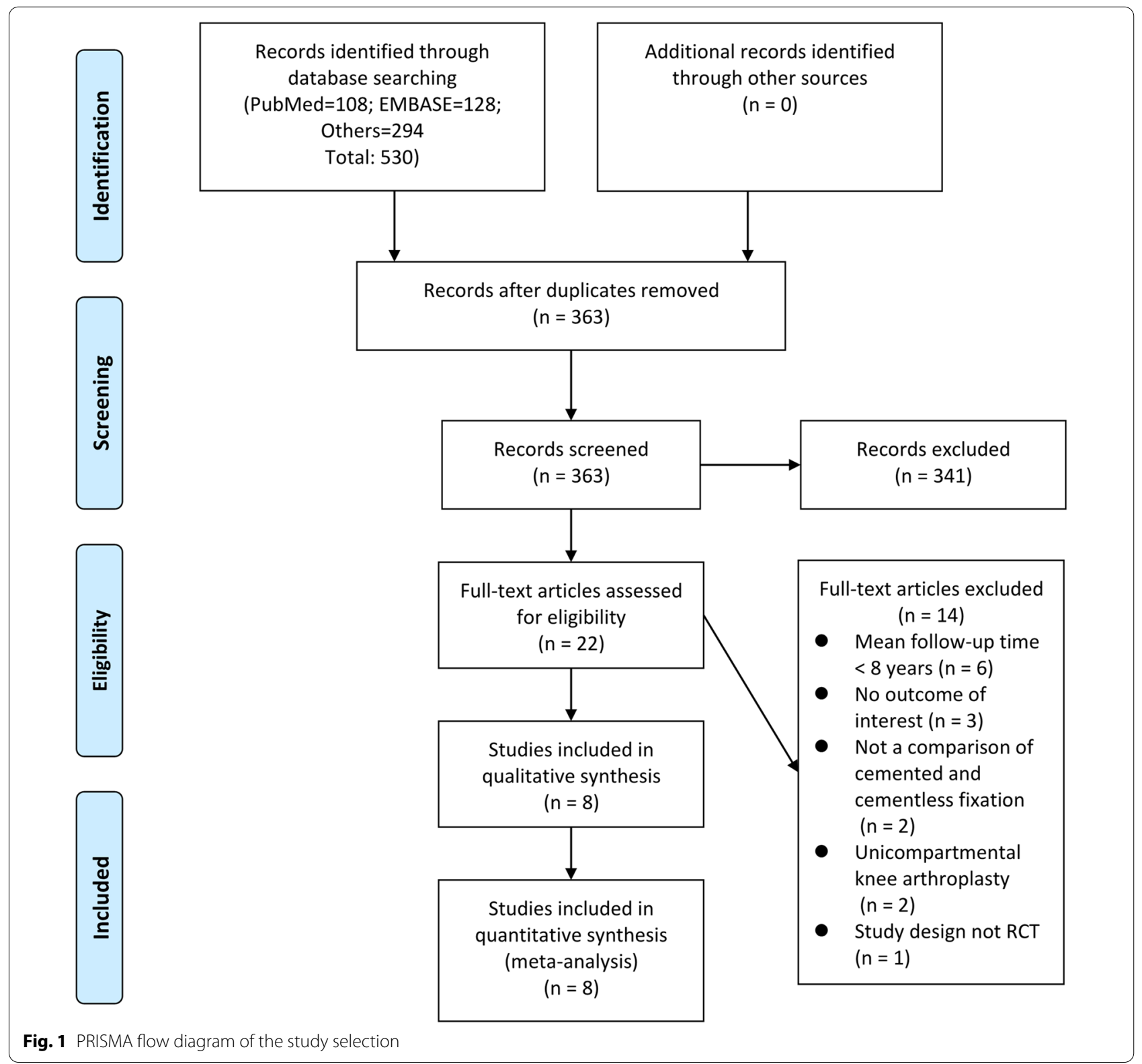

parable to the cemented fixation group (SMD - 0.065; $95 \% \mathrm{CI}-0.238$ to $0.109 ; P=0.463$ ) (Fig. 4B).

$K S$ pain score We assessed the $\mathrm{KS}$ pain score of cemented $(n=68)$ and cementless $(n=66)$ fixation of TKA by grouping together the KS pain score at last follow-up in 2 studies [27, 31]. After a fixed effect model $\left(I^{2}=2.5 \%\right)$ was applied, the pooled data revealed that the KS pain scores of the cementless fixation group of patients at $\geq 8.8$ years of follow-up were comparable to those of the cemented fixation group (SMD - 0.300; $95 \% \mathrm{CI}-0.641$ to $0.042 ; P=0.085$ ) (Fig. $4 \mathrm{C}$ ).
WOMAC score We assessed the WOMAC score of cemented $(n=216)$ and cementless $(n=212)$ fixation of TKA by grouping together the WOMAC score at last follow-up in 3 studies $[27,29,30]$. After the application of the fixed effect model $\left(I^{2}=13.6 \%\right)$, the pooled data revealed that the WOMAC scores of the cementless fixation group of patients at a minimum follow-up time of 8.8 years was comparable to the cemented fixation group (SMD - 0.117; $95 \% \mathrm{CI}-0.307$ to $0.073 ; P=0.227$ ) (Fig. 5A).

HSS score We assessed the HSS score of cemented $(n=110)$ and cementless $(n=126)$ fixation of TKA by 


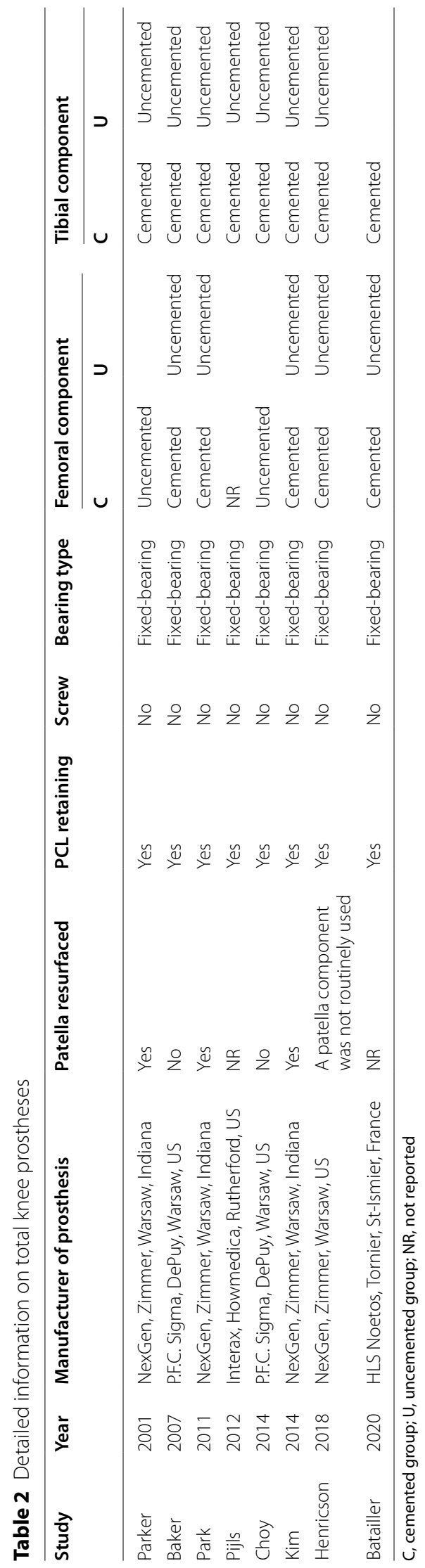


A

Random sequence generation (selection bias) Allocation concealment (selection bias)

Blinding of participants and personnel (performance bias)

Blinding of outcome assessment (detection bias)

Incomplete outcome data (attrition bias)

Selective reporting (reporting bias)

Other bias

\begin{tabular}{lllll}
\hline $0 \%$ & $25 \%$ & $50 \%$ & $75 \%$ & $100 \%$
\end{tabular}

Low risk of bias

Jnclear risk of bias

High risk of bias

B

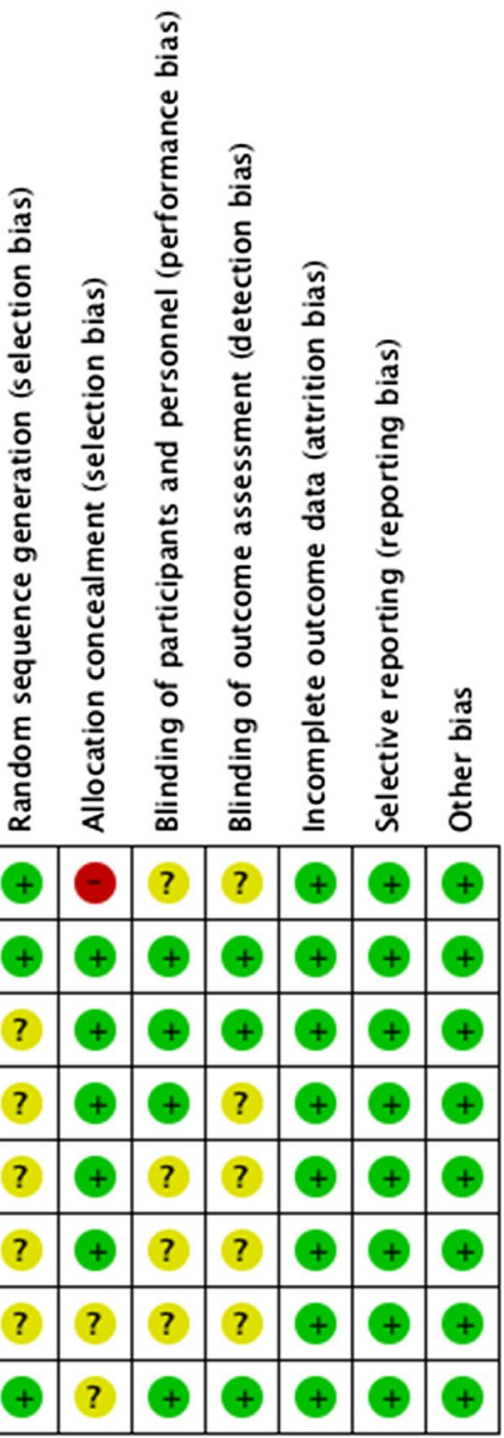

Fig. 2 Risk of bias (A) graph and (B) summary showing the authors'judgments about each risk of bias item presented as percentages across the 8 included RCTs 


Study
RR $(95 \% \mathrm{Cl})$

grouping together the HSS score at last follow-up in 2 studies [28, 29]. After using the fixed effect model $\left(I^{2}=0 \%\right)$, the pooled data revealed that the HSS scores of the cementless fixation group at a minimum 8.8 years of follow-up were comparable to those of the cemented fixation group of patients (SMD $-0.027 ; 95 \% \mathrm{CI}-0.270$ to 0.217; $P=0.829$ ) (Fig. 5B).

\section{ROM}

We assessed the ROM (in degree) of cemented ( $n=317)$ and cementless $(n=331)$ fixation of TKA by grouping together ROM at last follow-up in 6 studies [27-32]. Due to significant heterogeneity when using fixed model $\left(I^{2}>50 \%\right)$, we applied the random effect model. The pooled data revealed that the ROM of the cementless fixation group at minimum 8.8 years of follow-up was comparable to that of the cemented fixation group (SMD $0.061 ; 95 \% \mathrm{CI}-0.205$ to $\left.0.327 ; P=0.652, I^{2}=63.4 \%\right)$. We hypothesized that the inclusion of hybrid fixation might be a source of heterogeneity and did subgroup analysis of full cemented $v$ f full cementless group and full cemented/ cementless $v s$ hybrid group. Similar results were also seen in the subgroup analysis (Fig. 5C).

\section{Radiographic outcomes Incidence of radiolucent line}

We assessed the incidence of radiolucent line of cemented $(n=299)$ and cementless $(n=315)$ fixation of TKA by grouping together the incidence of radiolucent line at last follow-up in 5 studies [27-30, 32]. Due to significant heterogeneity $\left(I^{2}=57.7 \%\right)$, we applied the random effect model. Pooled data suggested that the incidence of the radiolucent line in the cementless fixation group of patients at a minimum of 8.8 years of follow-up was lower than that of the cemented fixation group (SMD 3.828; 95\% CI 2.228 to 6.576; $P<0.001$ ). We did a subgroup analysis according to the width of radiolucent line and found the heterogeneity greatly decreased. The incidence of radiolucent line $<1 \mathrm{~mm}$ in the cementless fixation group is significantly lower than that of the cemented fixation group (SMD 2.718; 95\% CI 1.818 to $4.065 ; P<0.001)$. A similar result was also observed in the subgroup of radiolucent line $\leq 2 \mathrm{~mm}$ (SMD 10.053; 95\% CI 3.829 to $26.395 ; P<0.001$ ) (Fig. 6A).

\section{MTPM}

We assessed the MTPM $(\mathrm{mm})$ of cemented $(n=42)$ and cementless $(n=60)$ fixation of TKA by grouping together the MTPM at last follow-up in 2 studies [28, 31]. Due to significant heterogeneity $\left(I^{2}=74.6 \%\right)$, the random effect model was employed. The pooled data strongly suggested that the MTPM of the cementless fixation group at a minimum 8.8 years follow-up was significantly greater than that of the cemented fixation group (SMD -0.739 ; $95 \% \mathrm{CI}-1.474$ to $-0.005 ; P=0.048$ ) (Fig. $6 \mathrm{~B}$ ). 


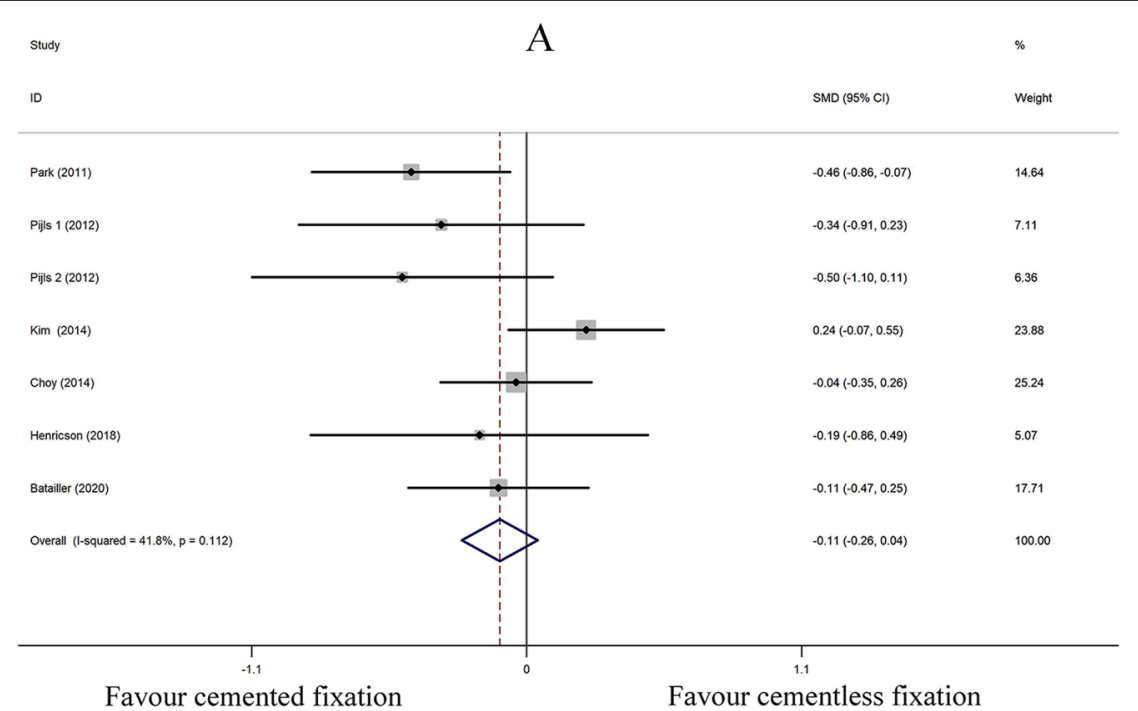

Favour cemented fixation

Favour cementless fixation
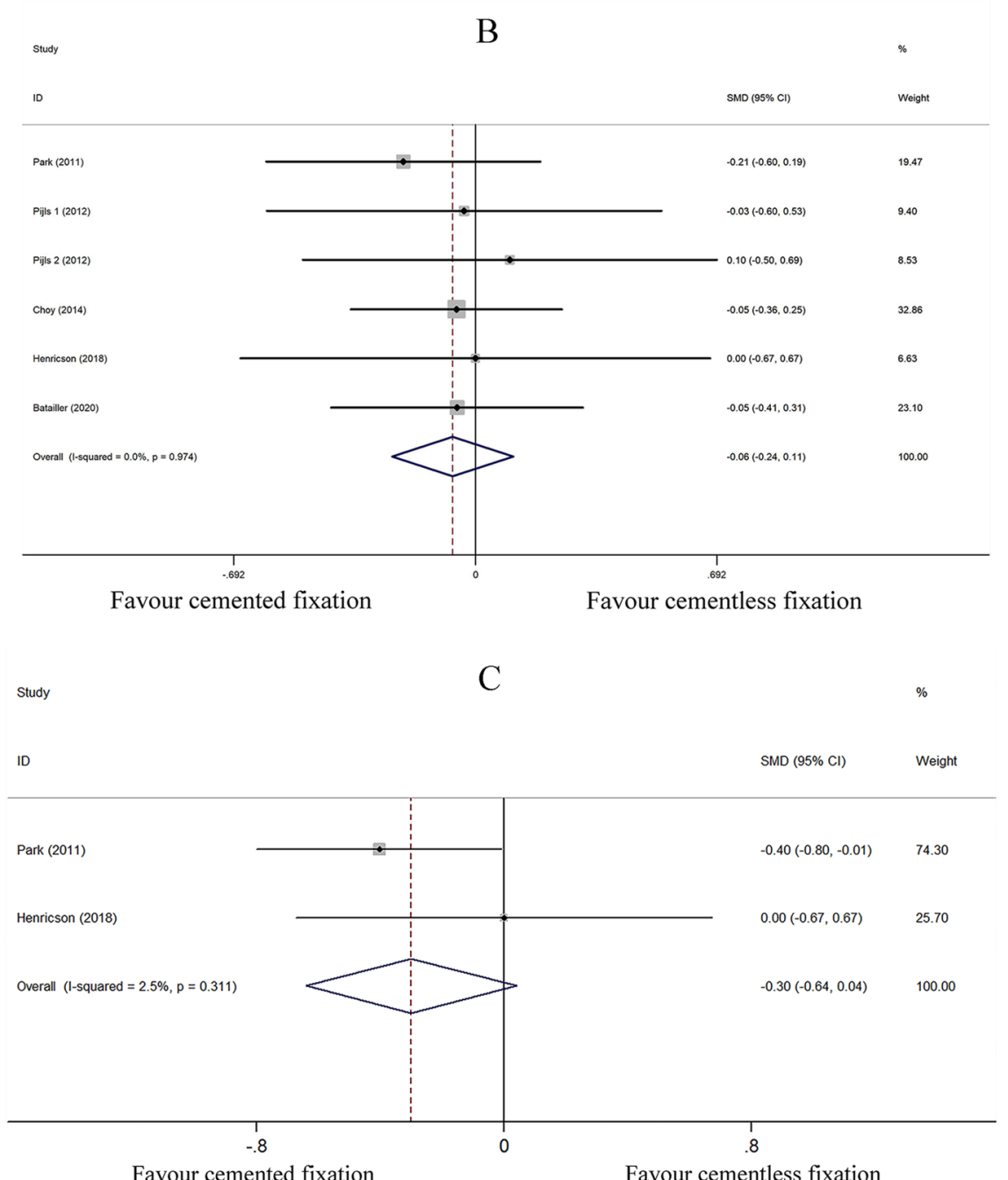

Fig. 4 Comparison analysis of (A) Knee Society (KS) knee score (B) KS function score (C) KS pain score in the cemented and cementless fixation groups 


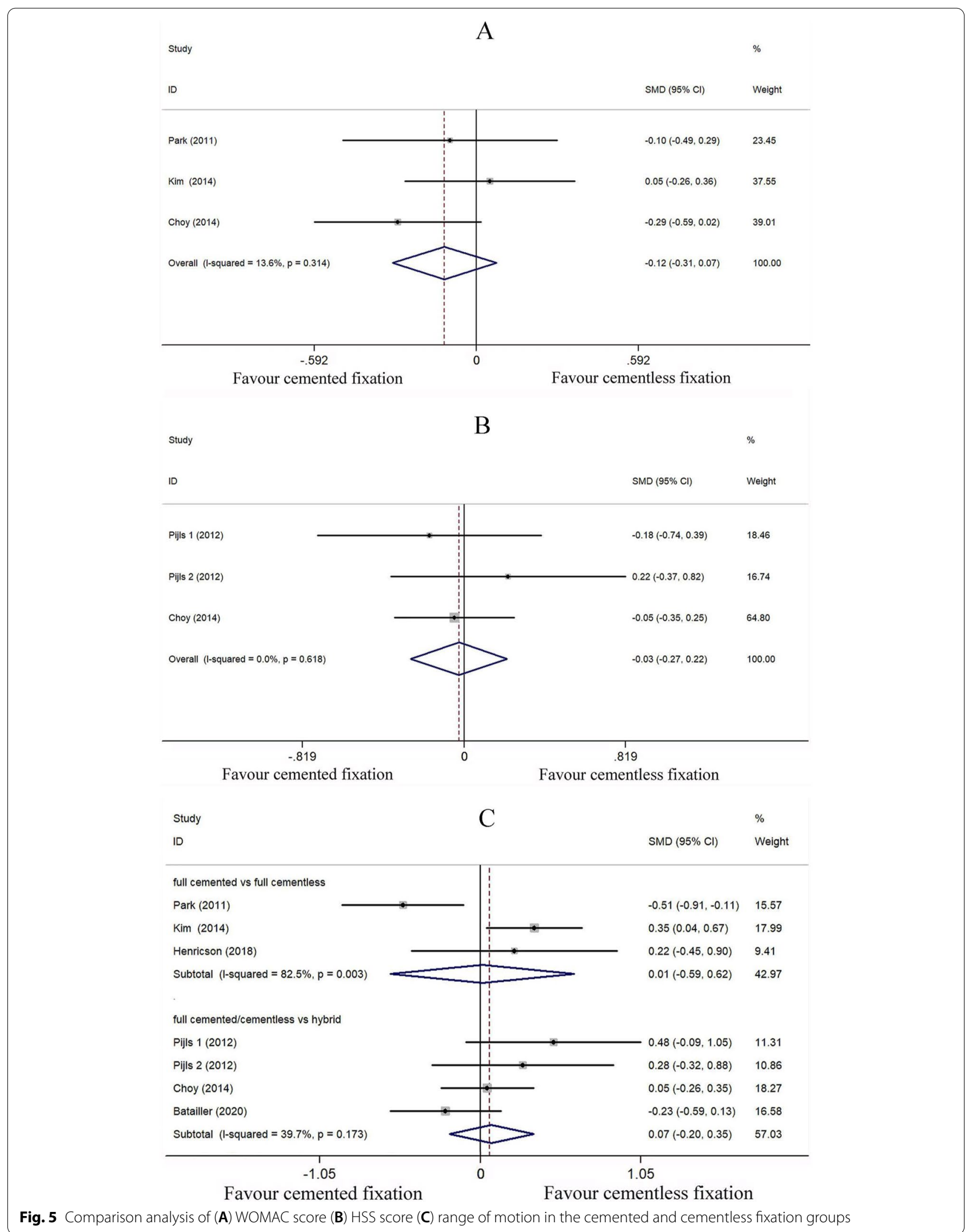




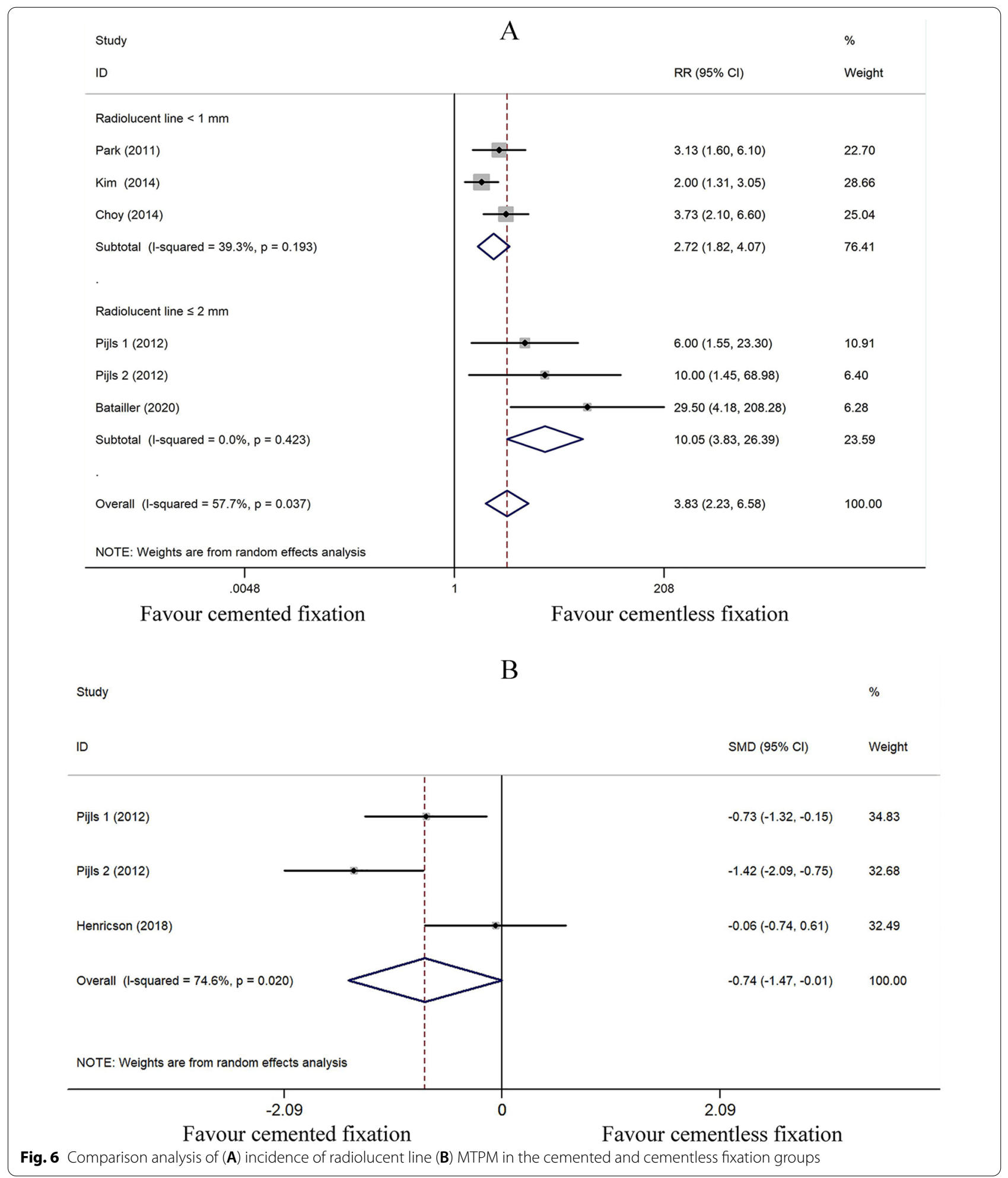

\section{Discussion}

A systematic review and meta-analysis of 8 RCT studies was carried out to investigate contemporary research results of the highest level of evidence for cemented or cementless fixation of TKAs. The available long-term evidence overwhelmingly indicated that cementless and cemented fixation have similar prosthesis survival rate, clinical scores, and mobility. However, cementless 
fixation causes less incidence of radiolucent line and more migration compared to cemented design.

Long term fixation is essential for cemented and cementless TKA, so the survival rate of prosthesis after TKA is one of the most important evaluation criteria in the clinical application of TKA [22, 24]. According to the results of previous studies, cemented fixation has advantages in early fixation and strength, while cementless fixation fails to show the same survival rate $[14,15]$. In the meta-analysis published in 2009, Gandhi et al. [19] found that the failure rate of cementless fixation was higher by analyzing RCTs and observational studies. However, with the sophisticated development of prosthesis manufacturing technology, in contrast to previous research, recent reports $[18,21$, 23] have shown that the survival rate of cementless prosthesis was similar to that of cemented prosthesis in short-term and medium-term follow-ups. Longterm follow-ups of TKA are essential before conclusions are made on the method of prosthesis fixation. Since most arthroplasty failures usually start near the 10-year mark [17], the significance of studies that have achieved good results in the short and mediumterm follow-up is questionable in assessing prosthesis longevity. Therefore, it is necessary and worthwhile to obtain long-term follow-up data for a more complete understanding of both fixed conditions. In the 8 RCTs included in this meta-analysis, the minimum followup period was 8.8 years, and we found that the survival rate of cementless design was not inferior to the cemented design in the long-term follow-up. The possible reason is that the new cementless prosthesis design and improved prosthesis surface treatment increase the friction between prosthesis and bone tissue and allow better bone-implant biocompatibility, which is conducive to bone formation and bone growth, and can maintain long-term permanent fixation [20].

In our study, KS knee scores, KS function scores, WOMAC scores, HSS scores, and ROM were used to evaluate functional recovery after a TKA procedure. Our meta-analysis revealed that there were no significant differences between the 2 fixation groups at the last follow-up time. About $50 \%$ of TKA patients experienced moderate to severe postoperative pain [33], so pain control after TKA is an important clinical issue. The cementless group had no obvious advantage over the cemented group in terms of KS pain score. Due to the small heterogeneity of the included studies in clinical scores and the pooled analyses were based on relatively large group of 134 to 615 participants, it is reliable to conclude that similar good clinical and functional improvements were found in the two techniques after a minimum 8.8 years follow-up.
The presence and size of radiolucent lines as introduced by the Knee Society were described to assess the potential degree of osteolytic lesions. Radiolucent lines are likely associated with loosening or instability of the implant, including migration and inadequate load distribution. In the present study, the pooled analysis of the incidence of radiolucent lines, based on a large group of 614 patients, indicated that cementless fixation was linked with better long-term radiological outcomes compared to the cemented patient group. In the subgroup analysis, similar results were observed in both radiolucent line $<1 \mathrm{~mm}$ and radiolucent line $<2 \mathrm{~mm}$ groups with acceptable heterogeneity.

Radio stereometric analysis (RSA) offers an in vivo method of studying fixation. Five to 10 or more years of RSA-data may be more reliable to predict long-term fixation, since different types of fixation design may differ in their pattern of migration [28]. The pooled analysis of MTPM at minimum 8.8 years after operation demonstrated less component migration in the cemented group compared with cementless design. It is well acknowledged that cemented implants can initially achieve more stable fixation owing to its distinct migration patterns with cementless implants [34]. It seems that from a long-term perspective, cemented implants also show less migration. However, due to high heterogeneity $\left(I^{2}=74.6 \%\right)$, the result should be viewed with a degree of caution.

Despite the strict inclusion criteria of this study, there are still some limitations: First, half of the RCTs of hybrid fixation (not full cemented or cementless components) were included in our study, which may have introduced potential bias in selection. Second, over half of the studies didn't report blinding of patients or outcome assessors, although blinding is often a problem in surgical trials [16], it may bias the meta-analysis. In addition, the identified language of only English and Chinese made publication bias inextricably.

\section{Conclusions}

Similar survival rates and clinical performance were observed for both cemented and cementless TKAs at a minimum 8.8 years of follow-up. However, radiography suggests each has its advantage in radiolucent line and MTPM. It seems both designs could be acceptable options for primary TKA in the long-term perspective. In the future, it is urgent to conduct researches on the bone condition and demographic characteristics of patients to help surgeons select the most suitable fixation method for patients. 


\section{Abbreviations}

HA: Hydroxyapatite; HSS: Hospital for special surgery; KS: Knee society; MTPM: Maximum total point motion; OA: Osteoarthritis; PCL: Posterior cruciate ligament; RA: Rheumatoid arthritis; RCT: Randomized controlled trials; ROM: Range of motion; RR: Relative risk; RSA: Radio stereometric analysis; SMD: Standardized mean difference; TKA: Total knee arthroplasty; WOMAC: Western Ontario and McMaster Universities Osteoarthritis Index.

\section{Supplementary Information}

The online version contains supplementary material available at https://doi. org/10.1186/s13018-021-02762-2.

Additional file 1. Table S1: MEDLINE search strategy. Figure S1: Egger's test survivorship. Figure S2: Egger's test KS knee score. Figure S3: Egger's test KS function score. Figure S4: Egger's test WAMAC score. Figure S5: Egger's test HSS score. Figure S6: Egger's test Range of motion. Figure S7: Egger's test radiolucent line. Figure S8: Egger's test MTPM.

\section{Acknowledgements}

Not applicable.

\section{Authors' contributions}

CC, YS, ZW and ZG were responsible for the conception and design of the study; CC, YS, ZW, ZG, CG and XB were responsible for acquisition of data; CC, YS, ZW, YC, CG and XB were responsible for analysis of data; CC and YS drafted the manuscript; CC, YS, ZG and YC were in charge of statistical analysis; all authors read and approved the final version of the manuscript.

\section{Funding}

None.

\section{Availability of data and materials}

All data generated or analysed during this study are included in this published article and its Additional files.

\section{Declarations}

Ethics approval and consent to participate

Not applicable.

\section{Consent for publication}

Not applicable.

\section{Competing interests}

The authors declare that they have no competing interests.

\section{Author details}

'Department of Orthopedics, Nanjing Lishui People's Hospital, Zhongda Hospital Lishui Branch, Southeast University, No. 86 Chongwen Road, Lishui District, Nanjing 211200, China. ${ }^{2}$ Department of Geriatrics, Kong Jiang Hospital of Yangpu District, Shanghai, China.

\section{Received: 14 March 2021 Accepted: 30 September 2021}

Published online: 12 October 2021

\section{References}

1. Phillips AM, Goddard NJ, Tomlinson JE. Current techniques in total knee replacement: results of a national survey. Ann R Coll Surg Engl. 1996;78(6):515-20.

2. Dahl OE. Cardiorespiratory and vascular dysfunction related to major reconstructive orthopedic surgery. Acta Orthop Scand. 1997;68(6):607-14.

3. Pagani $\mathrm{CH}$, Böhle C, Potthast W, Brüggemann GP. Short-term effects of a dedicated knee orthosis on knee adduction moment, pain, and function in patients with osteoarthritis. Arch Phys Med Rehabil. 2010;91(12):1936-41.

4. Sorrells RB, Murphy JA, Sheridan KC, Wasielewski RC. The effect of varus and valgus deformity on results of cementless mobile bearing TKA. Knee. 2007;14(4):284-8.

5. Søballe K, Hansen ES, Brockstedt-Rasmussen H, Bünger C. Hydroxyapatite coating converts fibrous tissue to bone around loaded implants. J Bone Joint Surg Br. 1993;75(2):270-8.

6. Bobyn JD, Stackpool GJ, Hacking SA, Tanzer M, Krygier JJ. Characteristics of bone ingrowth and interface mechanics of a new porous tantalum biomaterial. J Bone Joint Surg Br. 1999;81(5):907-14.

7. Nakama GY, Peccin MS, Almeida GJ, Lira Neto Ode A, Queiroz AA, Navarro RD. Cemented, cementless or hybrid fixation options in total knee arthroplasty for osteoarthritis and other non-traumatic diseases. Cochrane Database Syst Rev. 2012;10:Cd006193.

8. Zhou K, Yu H, Li J, Wang H, Zhou Z, Pei F. No difference in implant survivorship and clinical outcomes between full-cementless and fullcemented fixation in primary total knee arthroplasty: a systematic review and meta-analysis. Int J Surg. 2018;53:312-9.

9. Prasad AK, Tan JHS, Hanna SA, Dawson-Bowling S, Bedair HS. Cemented vs. cementless fixation in primary total knee arthroplasty: a systematic review and meta-analysis. EFORT Open Rev. 2020;5(11):793-8.

10. Chen C, Li R. Cementless versus cemented total knee arthroplasty in young patients: a meta-analysis of randomized controlled trials. J Orthop Surg Res. 2019;14(1):262.

11. Wang K, Sun H, Zhang K, Li S, Wu G, Zhou J, et al. Better outcomes are associated with cementless fixation in primary total knee arthroplasty in young patients: a systematic review and meta-analysis of randomized controlled trials. Medicine (Baltimore). 2020;99(3):e18750.

12. Wang C, Pfitzner T, von Roth P, Mayr HO, Sostheim M, Hube R. Fixation of stem in revision of total knee arthroplasty: cemented versus cementless-a meta-analysis. Knee Surg Sports Traumatol Arthrosc. 2016;24(10):3200-11.

13. Kurtz SM, Lau E, Ong K, Zhao K, Kelly M, Bozic KJ. Future young patient demand for primary and revision joint replacement: national projections from 2010 to 2030. Clin Orthop Relat Res. 2009:467(10):2606-12.

14. Berger RA, Lyon JH, Jacobs JJ, Barden RM, Berkson EM, Sheinkop MB, et al. Problems with cementless total knee arthroplasty at 11 years followup. Clin Orthop Relat Res. 2001;392:196-207.

15. Carlsson A, Björkman A, Besjakov J, Onsten I. Cemented tibial component fixation performs better than cementless fixation: a randomized radiostereometric study comparing porous-coated, hydroxyapatite-coated and cemented tibial components over 5 years. Acta Orthop. 2005:76(3):362-9.

16. Devereaux PJ, McKee MD, Yusuf S. Methodologic issues in randomized controlled trials of surgical interventions. Clin Orthop Relat Res. 2003:413:25-32.

17. Fernandez-Fairen M, Hernández-Vaquero D, Murcia A, Torres A, Llopis R. Trabecular metal in total knee arthroplasty associated with higher knee scores: a randomized controlled trial. Clin Orthop Relat Res. 2013;471(11):3543-53.

18. Fricka KB, Sritulanondha S, McAsey CJ. To cement or not? Two-year results of a prospective, randomized study comparing cemented vs. cementless total knee arthroplasty (TKA). J Arthroplasty. 2015;30(9 Suppl):55-8.

19. Gandhi R, Tsvetkov D, Davey JR, Mahomed NN. Survival and clinical function of cemented and uncemented prostheses in total knee replacement: a meta-analysis. J Bone Joint Surg Br. 2009;91(7):889-95.

20. Gerscovich D, Schwing C, Unger A. Long-term results of a porous tantalum monoblock tibia component: clinical and radiographic results at follow-up of 10 years. Arthroplast Today. 2017;3(3):192-6.

21. Hu B, Chen Y, Zhu H, Wu H, Yan S. Cementless porous tantalum monoblock tibia vs cemented modular tibia in primary total knee arthroplasty: a meta-analysis. J Arthroplasty. 2017;32(2):666-74.

22. Mont MA, Pivec R, Issa K, Kapadia BH, Maheshwari A, Harwin SF. Longterm implant survivorship of cementless total knee arthroplasty: a systematic review of the literature and meta-analysis. J Knee Surg. 2014;27(5):369-76.

23. Schotanus MGM, Pilot P, Kaptein BL, Draijer WF, Tilman PBJ, Vos R, et al. No difference in terms of radiostereometric analysis between fixed-and mobile-bearing total knee arthroplasty: a randomized, single-blind, controlled trial. Knee Surg Sports Traumatol Arthrosc. 2017;25(9):2978-85. 
24. Wang H, Lou H, Zhang H, Jiang J, Liu K. Similar survival between uncemented and cemented fixation prostheses in total knee arthroplasty: a meta-analysis and systematic comparative analysis using registers. Knee Surg Sports Traumatol Arthrosc. 2014;22(12):3191-7.

25. Parker DA, Rorabeck CH, Bourne RB. Long-term followup of cementless versus hybrid fixation for total knee arthroplasty. Clin Orthop Relat Res. 2001;388:68-76.

26. Baker PN, Khaw FM, Kirk LM, Esler CN, Gregg PJ. A randomised controlled trial of cemented versus cementless press-fit condylar total knee replacement: 15-year survival analysis. J Bone Joint Surg Br. 2007;89(12):1608-14.

27. Park JW, Kim YH. Simultaneous cemented and cementless total knee replacement in the same patients: a prospective comparison of longterm outcomes using an identical design of NexGen prosthesis. J Bone Joint Surg Br. 2011;93(11):1479-86.

28. Pijls BG, Valstar ER, Kaptein BL, Fiocco M, Nelissen RG. The beneficial effect of hydroxyapatite lasts: a randomized radiostereometric trial comparing hydroxyapatite-coated, uncoated, and cemented tibial components for up to 16 years. Acta Orthop. 2012;83(2):135-41.

29. Choy WS, Yang DS, Lee KW, Lee SK, Kim KJ, Chang SH. Cemented versus cementless fixation of a tibial component in LCS mobile-bearing total knee arthroplasty performed by a single surgeon. J Arthroplasty. 2014;29(12):2397-401.

30. Kim YH, Park JW, Lim HM, Park ES. Cementless and cemented total knee arthroplasty in patients younger than fifty five years. Which is better? Int Orthop. 2014;38(2):297-303.

31. Henricson A, Wojtowicz R, Nilsson KG, Crnalic S. Uncemented or cemented femoral components work equally well in total knee arthroplasty. Knee Surg Sports Traumatol Arthrosc. 2019;27(4):1251-8.

32. Batailler C, Malemo Y, Demey G, Kenney R, Lustig S, Servien E. Cemented vs uncemented femoral components: a randomized, controlled trial at 10 years minimum follow-up. J Arthroplasty. 2020;35(8):2090-6.

33. Bonica J, Loeser J, Chapman C, Fordyce W. The management of pain. 2nd ed. Philadelphia: Lea and Febiger; 1990.

34. Freeman MA, Tennant R. The scientific basis of cement versus cementless fixation. Clin Orthop Relat Res. 1992;276:19-25.

\section{Publisher's Note}

Springer Nature remains neutral with regard to jurisdictional claims in published maps and institutional affiliations.
Ready to submit your research? Choose BMC and benefit from:

- fast, convenient online submission

- thorough peer review by experienced researchers in your field

- rapid publication on acceptance

- support for research data, including large and complex data types

- gold Open Access which fosters wider collaboration and increased citations

- maximum visibility for your research: over 100M website views per year

At BMC, research is always in progress.

Learn more biomedcentral.com/submissions 